\title{
Propuesta de un modelo educativo que permita mejorar la satisfacción estudiantil en la Facultad de Ingeniería de la Universidad Privada de Tacna
}

\author{
Proposal for an educational model that enables improve student satisfaction in the Faculty of Engineering of the Private \\ University in Tacna

\section{Martha Judith Paredes Vignola ${ }^{1}$}

\begin{abstract}
RESUMEN
Objetivo: Elevar el número de estudiantes y mejorar las oportunidades laborales de los egresados.

Método: Se propone un modelo educativo "de gestión de calidad educativa" que permita mejorar la satisfacción estudiantil en la Facultad de Ingeniería de la Universidad Privada de Tacna, que permita establecer un proceso de mejora continua a través de la medición y evaluación del logro de aprendizajes.

Resultados: En gestión de la calidad se presenta una información consolidada al respecto, obteniéndose un valor promedio general de 192,93 con una desviación típica de 23,239; que al ubicarlo en la escala de valoración predeterminada. En la satisfacción estudiantil se encontró que el valor medio de las expectativas fue de 87,04 que al ubicarlo en la escala de valoración previamente definida cae en la zona de "Expectativa de FAING adecuada"; el valor medio de las percepciones fue de 73,40 que implica que cae en la zona de "Percepción de FAING adecuada". La expectativa y percepciones del estudiante se aprecian de cada una de las respuestas globales obtenidas referida a la satisfacción o insatisfacción estudiantil con una media de -13.64 y desviación típica de 25.798, que implica que la media cae debajo de la zona de "Expectativa (Percepción) de FAING muy inadecuada". Conclusión: Se concluye que a medida que los docentes tienen más años de experiencia académica consideran que la gestión de calidad educativa no está mejorando de acuerdo a las exigencias del entorno laboral y de las expectativas de los estudiantes, que existe un nivel de insatisfacción estudiantil por la calidad de la enseñanza recibida en la FAING - UPT, a pesar de que consideran que el nivel educativo es adecuado, concluyendo que es la EP de Ing. Agroindustrial en donde existe un mayor nivel de insatisfacción.
\end{abstract}

\section{PALABRACLAVE}

Modelo educativo, satisfacción estudiantil, objetivos educativos del programa, resultados de aprendizaje, evaluación.

\begin{abstract}
Objective: Increasing the number of students and improve employment opportunities of graduates.

Method: Proposes an educational model "of educational quality management" that allows to improve student satisfaction in the Faculty of Engineering of the Private University of Tacna, that allows to establish a process of continuous improvement through measurement and evaluation of learning achievement.
\end{abstract}

Results: Quality Management we present consolidated information about it, obtaining an overall average value of 192.93 with a standard deviation of 23,239 that by placing it in the default rating scale. In student satisfaction was found that the average value was 87.04 expectations that locate in the grading scale previously defined area falls "suitable FAING expectancy", the average value was 73 perceptions, 40 implies that falls in the zone of "proper FAING Perception". The expectation and student perceptions appreciate each of global responses obtained referring to student satisfaction or dissatisfaction with a mean of 13.64 and typical deviation of 25,798, which implies that the mean falls below the zone of Expectation (Perception) of FAING very inadequate.

Conclusion: We conclude that as teachers have more years of academic experience management believe that the quality education is not improving according to the demands of the work environment and the expectations of students, there is a level of student dissatisfaction by the quality of education received by the FAING- UPTdespite they consider that the educational level is adequate, concluding that EP is the Engineering Agroindustrial where there is a high level of dissatisfaction.

\section{KEYWORDS}

Educational model, student satisfaction, program educational objectives, learning outcomes, evaluation.

1. Doctor en Educación con mención en Gestión Educativa, Magister en Informática e Ingeniero de Sistemas. Actualmente Labora como Jefe de la Oficina de Computo de la Universidad Privada de Tacna. Especializada en gestión de proyectos de TI, Gestión de calidad académica, Certificado en ITIL, Evaluador externo del CONEAU.

E-mail: martijudi@hotmail.com 


\section{Introducción}

Un modelo educativo se proyecta hacia el logro de estándares de calidad en la gestión de la educación superior, al potenciar la investigación científica, la innovación tecnológica y empresarial, y el desarrollo de la "condición humana" dentro de la sociedad.

Para abordar la tarea de calidad en la educación se presentan dos opciones: se puede diseñar un modelo de calidad propio o se puede optar por adecuarse a un modelo determinado, por lo cual los modelos sirven para evaluar el nivel de calidad de una institución educativa que decide presentar a la sociedad sus logros. Un modelo por ser una representación de la realidad se puede adecuar al contexto que deseamos representar.

La Facultad de Ingeniería ha emprendido acciones para evaluar y garantizar la calidad del servicio educativo. Por ello, para fortalecer estas acciones se presenta la propuesta de un modelo educativo de gestión de calidad educativa que permita mejorar la satisfacción estudiantil de la Facultad de Ingeniería de la Universidad Privada de Tacna con el objetivo elevar la cantidad de estudiantes y mejorar las oportunidades laborales de sus egresados.

Este trabajo de investigación es descriptivo puesto que se describieron los hechos como son observados y explicativos, puesto que se buscó analizar las relaciones de causa-efecto de la satisfacción estudiantil que permita proponer un modelo educativo.

Pregunta: La pregunta de investigación se formula de la siguiente manera: ¿Cómo un modelo educativo propuesto de Gestión de calidad educativa permitirá mejorar la satisfacción estudiantil de la Facultad de Ingeniería de la Universidad Privada de Tacna?

Justificación: La Facultad de Ingeniería para evaluar y garantizar la calidad del servicio educativo ha emprendido acciones en vías de la autoevaluación y acreditación, por ello para fortalecer estas acciones se presenta la propuesta de un modelo educativo.

Para realizar esta propuesta se ha considerado los siguientes criterios:

- Las acciones emprendidas por la Facultad de Ingeniería para evaluar y garantizar la calidad de la educación.

- Ausencia de mecanismo para medir y evaluar el servicio educativo.
- Proceso de mejora continua no establecido en la Facultad de Ingeniería.

- Competitividad entre universidades para captar más estudiantes.

- Pocas oportunidades laborales para los nuevos egresados de la Facultad de Ingeniería.

En base a lo señalado en los párrafos precedentes, y principalmente de acuerdo a mi experiencia docente, se justifica el presente trabajo de investigación buscando fortalecer la gestión de la Facultad de Ingeniería de la Universidad Privada de Tacna.

El modelo educativo diseñado considera procesos para gestionar una escuela profesional en aspectos como el planeamiento académico, la gestión de recursos, la gestión del proceso educativo enseñanza aprendizaje y la gestión del proceso de mejora continua, estos procesos fluyen en un ciclo continuo de mejora continua. Estaré centrando el desarrollo del modelo en el subproceso de Mejora Continua por no estar establecido en la Facultad.

El Subproceso de Mejora Continua permite evaluar el aprendizaje de los cursos de los estudiantes, evaluar los resultados de aprendizaje del estudiante al finalizar la carrera y evaluar el logro de los objetivos educativos en el desempeño profesional del egresado después de cierto tiempo de haber culminado la carrera, dentro de un cronograma establecido, aplicando una serie de herramientas de medición cuyos resultados serán analizados para determinar acciones de mejora en función al logro de los resultados de aprendizaje así como de los objetivos educativos de la carrera.

Las condiciones básicas que debería tener la Facultad de Ingeniería para que tenga éxito el modelo si fuese aplicado serían:

- Apoyo de la alta dirección

- Trabajo en equipo

- Personal capacitado para participar en el proceso

- Presupuesto adecuado para cubrir el costo del proyecto

- Disposición al cambio de parte de docentes, estudiantes, administrativos y autoridades

- Compromiso del personal que participa en el Proyecto 
Para el diseño de la propuesta del Modelo Educativo para la Facultad de Ingeniería de la UPT, se hará uso de la notación BPMN para representar el proceso de negocio de la Universidad, por ser un estándar internacional de modelado de procesos aceptado por la comunidad, la cual permite modelar los procesos de una manera unificada permitiendo un entendimiento a todas las personas de una organización. Business Process Modeling Notation (BPMN) es una notación gráfica que describe la lógica de los pasos de un proceso de Negocio. Proporciona un lenguaje común para que las partes involucradas puedan comunicar los procesos de forma clara, completa y eficiente. De esta forma BPMN define la notación y semántica de un Diagrama de Procesos de Negocio (Business Process Diagram, BPD).

Utilizando la notación BPMN modelaríamos el Modelo Educativo del Sistema de Gestión de Calidad Educativa, que está representado en la figura $\mathrm{N}^{0} 1$ :

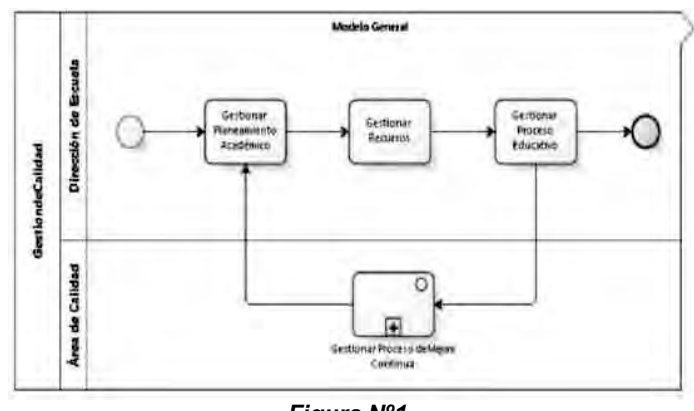

Sistema de gestión de calidad educativa (BPMN)

- En el proceso "Gestionar Planeamiento Académico" se planifica las actividades del inicio de cada ciclo académico, tales como: elaborar la carga horaria de docentes, los cursos a dictarse, horarios, proyecciones de alumnos matriculados, plan estratégico de la carrera, reuniones académicas, tutoría, convenios con empresas, proyectos de extensión y proyección social.

- En el proceso "Gestionar Recursos" se planifica la adquisición de todos los recursos necesarios para desarrollar las actividades del ciclo académico como materiales, equipos, aulas y laboratorios.

- En el proceso "Gestionar el Proceso Educativo Enseñanza Aprendizaje" se controla la ejecución del proceso educativo, a través del seguimiento del avance silábico, revisión de contenidos de sílabos, actualización de contenidos del plan de estudios, visitas a clases, seguimiento de prácticas pre profesionales, análisis de situación de egresados, entre otros.

- En el proceso "Gestionar el Proceso de Mejora Continua" se realiza el seguimiento y control de las actividades desarrolladas en el proceso educativo, con el fin de verificar que se cumple el nivel de calidad en la formación del estudiante. Así como la evaluación de resultados a través de herramientas de medición (assessment involucra la identificación, preparación y medición) que permitirán proporcionar resultados y acciones de mejora para que se implementen en el proceso educativo. A este proceso se le debe dar mayor atención puesto que es el punto principal a evaluar en que estamos fallando en el proceso educativo.

- El proceso "Gestionar Proceso de Mejora Continua" es a la vez un subproceso que contiene más información. Un subproceso es una actividad compuesta que es incluida dentro de un proceso, incluye un conjunto de actividades y una secuencia lógica que indica que dicha actividad puede ser analizada en más detalle (ver figura $\mathrm{N}^{0} 2$ ). Estaré centrando el modelo enfocado al sub Proceso de Mejora Continua por no estar establecido en la Facultad. Este proceso permite evaluar el aprendizaje de los cursos, evaluar los resultados de aprendizaje y evaluar el logro de los objetivos educativos dentro de un cronograma establecido, aplicando una serie de herramientas de medición cuyos resultados serán analizados para determinar acciones de mejora en función al logro de los resultados de aprendizaje así como de los objetivos educativos de la carrera.

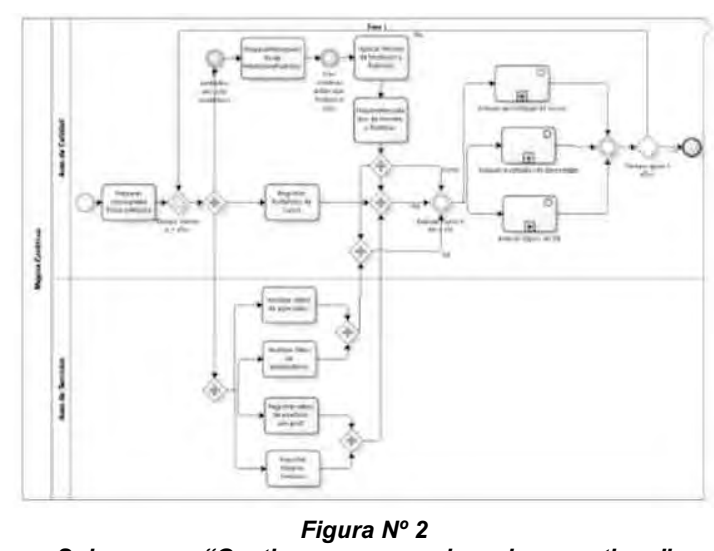

Subproceso "Gestionar proceso de mejora continua"

El evento de inicio comienza con la actividad "Preparar Cronograma del Proceso de Mejora", 
aquí se calendarizan las actividades de asessment en el transcurso de un año. La compuerta de inicio valida si nos encontramos dentro del tiempo, si es así se activa una compuerta paralela que permite activar varios caminos en paralelo. Entre estos caminos tenemos:

- La compuerta paralela puede activar el evento intermedio "A Mediados del Ciclo Académico" para continuar con la actividad preparar herramientas de medición. Después se activa el evento intermedio de "tres Semanas Antes que Finalice el Ciclo Académico" para continuar con el flujo a través de la actividad aplicar herramientas de medición y rubricas, y continuar con la actividad preparar resultados de herramientas 0 rúbricas. Las herramientas y rubricas se aplican en los cursos definidos por los evaluadores, y son cursos terminales 0 integradores de un determinado perfil. Estos procesos permiten capturar datos de encuestas, procesarlas y preparar un informe con el análisis de los resultados. Estas actividades los realiza el área de calidad de la Escuela Profesional y servirá para analizar un ciclo académico el logro de los cursos.

- La compuerta paralela también puede activar el proceso "Registrar el Portafolio de Cursos" que permite registrar los documentos que debe presentar el docente por cada unidad de aprendizaje del curso que enseña, los documentos pueden ser exámenes, prácticas calificadas, trabajos prácticos, apuntes de clase, laboratorios y otros en formato digital o papel, que servirán como evidencia para el análisis posterior, este proceso contribuye a evaluar los Resultados de Aprendizaje.

- Además, la compuerta puede activar otra paralela para realizar las actividades analizar datos de egresados y empleadores, registrar datos de prácticas pre profesionales 0 reportar mejoras a servicios educativos. Los datos de análisis de egresados y empleadores han sido tomados a partir de las encuestas realizadas por el área de calidad e información registrada en la base de datos de egresados y servirán para analizar los Objetivos Educativos.

- Los reportes de mejoras a servicios son el resultado del trabajo realizado en la escuela en la mejora de los servicios educativos que ofrece la escuela y la universidad basándose en las encuestas aplicadas de servicios. El registro de datos de prácticas pre profesionales son el resultado de las entrevistas de los supervisores de prácticas con los empleadores para conocer sobre el desempeño de los practicantes y servirán para analizar los Resultados de Aprendizaje.

- Los flujos generados por la primera compuerta pueden converger en una compuerta inclusiva de decisión lo que significa que esperará el primer camino que llegue para continuar con la siguiente actividad del flujo. Después de la espera y de evaluarse la condición de Curso o RA, OE se selecciona realizar uno de los sub procesos: evaluar el aprendizaje de los cursos, evaluar los resultados de aprendizaje o evaluar los logros de los objetivos educativos.

- Para evaluar la condición de Curso sólo se esperará a que llegue el flujo de la compuerta paralela que proviene de la actividad Preparar resultados de herramientas o rúbricas.

- Para evaluar la condición de RA, es necesario esperar a que llegue el flujo de la compuerta paralela que proviene de la actividad Preparar resultados de herramientas 0 rúbricas, registrar portafolio de cursos, registrar datos de prácticas pre profesionales y reportar mejora de servicios

- Para evaluar la condición OE, es necesario esperar a que llegue el flujo de la compuerta paralela que proviene de la actividad Preparar resultados de herramientas o rúbricas, analizar datos de egresados y analizar datos de empleadores.

- Por último se decide si se cumplió el tiempo de los 3 años si no es así se continua con el flujo en caso contrario finaliza el proceso.

\section{a) Subproceso "Evaluar el Aprendizaje de los Cursos"}

Para evaluar el logro del aprendizaje de los estudiantes en los cursos integradores, se activa el evento de inicio temporizador "Tres Semanas Antes que Finalice el Ciclo Académico" para continuar con la actividad preparar situación académica del estudiante que consiste en obtener reportes de notas de situación de estudiantes con alta, media y bajas calificaciones. 
Además, se activa el evento de inicio "A Mitad del Ciclo Académico" que indica que se deben reportar las visitas realizadas a las clases para evaluar el desempeño docente y atención de alumnos en las clases.

A la vez, se dispone de información del resultado de encuestas de cursos y portafolio de cursos, en la última semana del ciclo académico se produce el evento que inicia la actividad para registrar todos los informes de ejecución de cursos que son elaborados por los docentes para evaluar el progreso del proceso enseñanza aprendizaje a lo largo del ciclo académico, esta información es proporcionado por el área de calidad.

Después de estas actividades se evalúan los informes generados, reportes, portafolio, registros, rúbricas y encuestas en cursos integradores, se analiza si los resultados se encuentran por debajo de la meta si es así se proponen acciones de mejora, se validan las acciones de mejora propuestas, estos procesos son realizados por el Comité Académico de la Escuela Profesional.

La Dirección de la Escuela Profesional es la encargada de la actividad implementar las acciones de mejora en el transcurso de un ciclo académico después del cuál en reunión del comité académico se realiza la actividad verificar la eficacia de las acciones de mejora y finaliza con un evento de fin (Ver Figura $N^{\circ} 3$ ).

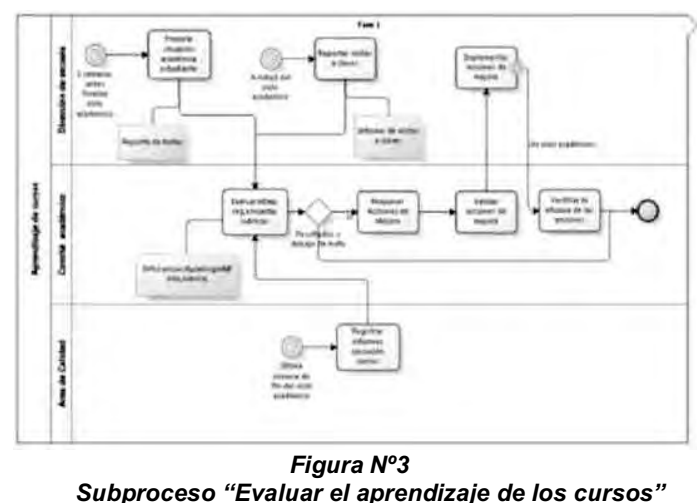

\section{b) Subproceso "Evaluar Resultados de Aprendizaje"}

Los resultados de aprendizaje se refieren al logro de las competencias del estudiante de los últimos ciclos académicos (IX y X ciclo).

El subproceso inicia con activación de evaluar informes, portafolio y resultados de encuestas. Las encuestas evalúan el nivel de logro de capacidades de los resultados de aprendizaje en los estudiantes. Las encuestas se aplican también para evaluar el logro de capacidades en los cursos de talleres integradores $y$ servicios educativos.

Después de evaluar toda la información, se realiza la actividad "Evaluar el Plan del Diseño Curricular" que consiste en revisar el plan de estudios, el perfil profesional, los silabos, contenidos de cursos y docentes. Se evalúa si se encuentran aspectos a mejorar, si es positivo se evalúa si es necesario actualizar el diseño curricular, si respondemos positivamente se activa el paso "Actualizar el Diseño Curricular" que será realizado por el Comité de Diseño Curricular y finaliza el proceso. En caso contrario se registran las acciones de mejora. Se aprueban las acciones de mejora a implementar por la Dirección de Escuela Profesional.

En forma paralela, la Dirección de la Escuela Profesional implementa las acciones de mejora en el transcurso de un año, mientras se activa el evento intermedio temporizador "Cada Trimestre" para realizar la actividad registrar resultados de las acciones de mejora implementadas. Además cada semestre se activa el evento "Intermedio Temporizador" para verificar la eficacia de los planes de mejora implementados, si el tiempo de implementación es menor a 1 año entonces el flujo regresa al evento intermedio "Cada trimestre" y continúa el flujo hasta que se cumpla la condición del tiempo de implementación de 1 año; es decir, después de un año evaluamos los resultados de aprendizaje (Ver figura 4).

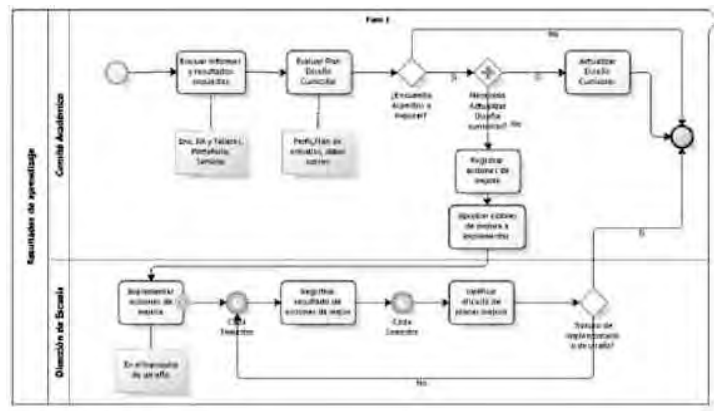

Figura $\mathrm{N}^{\circ} 4$

Subproceso "Evaluar resultado de aprendizaje"

c) Sub proceso "Evaluar Logros de Objetivos Educativos"

El proceso inicia cuando se evalúan los resultados de encuestas aplicadas a egresados y empleadores, perfiles profesionales $y$ resultados de aprendizaje, como resultado de este proceso se proponen cambios a los 
objetivos educativos analizando la opinión de los egresados respecto al logro de competencias profesionales en ellos mismos y a los empleadores sobre la satisfacción en el desenvolvimiento de las competencias profesionales de los egresados. A continuación, se validan los objetivos educativos con el fin de relacionarlos con los resultados del programa, la visión y misión de la Escuela Profesional y de la Facultad de Ingeniería de la UPT.

Si los objetivos educativos son validados se actualizan los objetivos educativos, en caso contrario finaliza el proceso. La actualización de los mismos, permitirá definir mejoras a los resultados de aprendizaje y esto, a la vez, conllevará a mejorar el diseño curricular, en el cuál se deberá actualizar el perfil profesional, plan de estudios, sílabos, etc. Estas actividades son realizadas por el Comité Consultivo.

La Dirección de la Escuela Profesional se encargará de implementar las acciones de mejora hasta por un período de tres años, a través del cual, cada medio año se procederá a registrar los resultados de acciones de mejora y cada año verificar la eficacia de las acciones de mejora. Si no se cumplen los tres años de cambios se activa nuevamente el evento intermedio temporizador "A mitad del año" y sigue la secuencia de procesos hasta que se cumple el plazo de 3 años para proceder a salir del proceso en el evento de fin de proceso(Ver Figura $\mathrm{N}^{\circ}$ ).

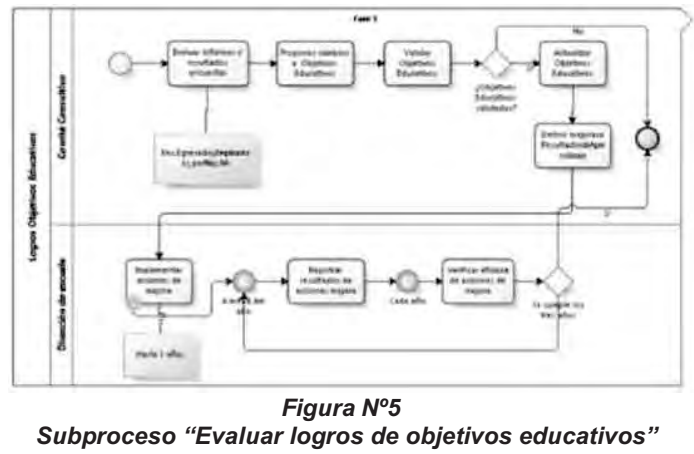

\section{Materiales y métodos}

La población fue de 983 alumnos y 88 docentes, la muestra de 146 alumnos y 58 docentes. (Referencia semestre académico 2010-I).

\section{Fuentes de Información}

La fuente de información fue primaria, puesto que se aplicaron los instrumentos a los estudiantes (de preferencia que tengan por lo menos 01 año en la FAING-UPT) y docentes (de preferencia que tengan por lo menos 02 años trabajando para la FAING-UPT).

\section{Tipo de investigación}

La presente investigación es descriptiva puesto que se describieron los hechos como son observados, y explicativa puesto que se buscó analizar las relaciones de causa-efecto de la satisfacción estudiantil para proponer un modelo educativo.

\section{Diseño de investigación}

El diseño de la investigación es de características no experimentales, cuya recolección de información fue de corte transversal.

\section{Hipótesis de Investigación}

El modelo educativo propuesto de gestión de calidad educativa permitirá mejorar la satisfacción estudiantil en la Facultad de Ingeniería de la Universidad Privada de Tacna. El análisis de la variable01 implicó una Escala de Valoración siendo sus niveles los siguientes:

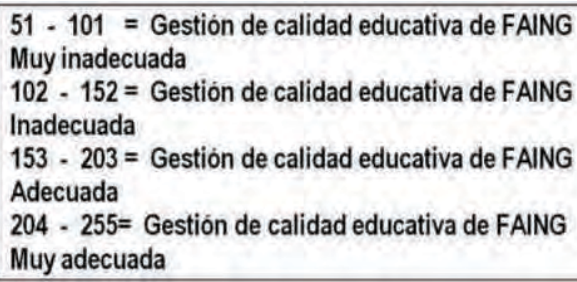

El análisis de la variable 02 implicó identificar una Escala de Valoración siendo sus niveles los siguientes:

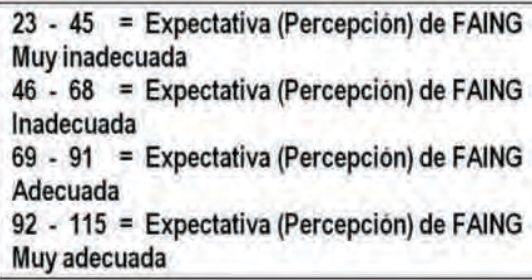

\section{Resultados}

Después de desarrollar un análisis de cada uno de los indicadores de la variable "Gestión de calidad educativa", se presenta una información consolidada al respecto, obteniéndose un valor promedio general de 192,93 con una desviación típica de 23,239; que al ubicarlo en la escala de valoración predeterminada, se concluye que la Gestión de calidad educativa en la Facultad de Ingeniería de la Universidad Privada de Tacna es ADECUADA (desde la perspectiva de los docentes). 


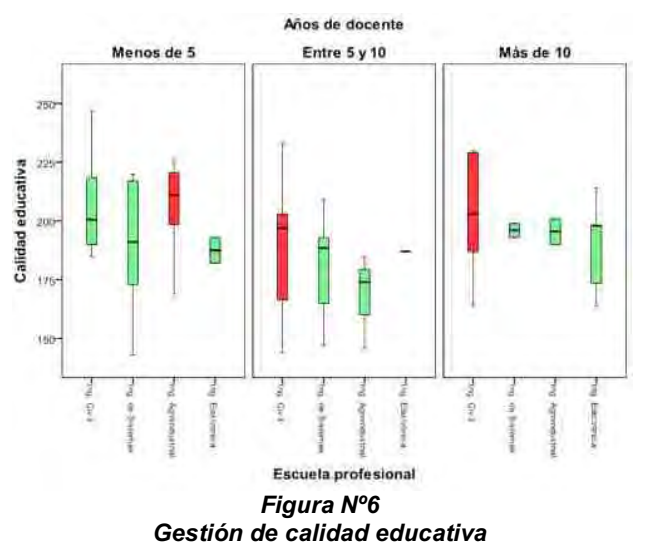

La Figura $N^{\circ} 6$ muestra que a medida que los docentes tienen más años de experiencia académica consideran que la gestión de calidad educativa no está mejorando de acuerdo a las exigencias del entorno laboral y de las expectativas de los estudiantes.

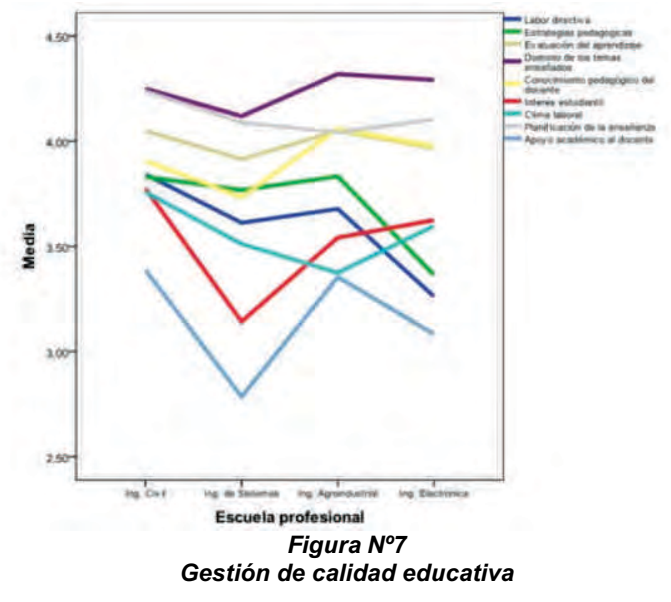

En la Figura $\mathrm{N}^{\circ} 7$ se muestra un análisis detallado, que permite concluir que los docentes de FAING consideran que los indicadores más destacados son: "Dominio de los temas enseñados" y "Planificación de la enseñanza"; y los indicadores a fortalecer son: "Apoyo académico al docente" y el "Interés estudiantil".

Para desarrollar el análisis global de la variable "Satisfacción estudiantil" se procedió a sumar todos los puntajes a las respuestas afines a las Expectativas y a las Percepciones de los estudiantes, referente al servicio educativo recibido por la FAING - UPT, obteniéndose la Tabla No 1:
Tabla No 1

Satisfacción Estudiantil

\begin{tabular}{|l|c|c|}
\hline & Media & Desv. típ. \\
\hline Expectativas & 87,04 & 24,183 \\
Percepciones & 73,40 & 18,944 \\
Satisfacción o & $-13,64$ & 25,798 \\
insatisfacción & & \\
\hline
\end{tabular}

Se encontró que el valor medio de las expectativas fue de 87,04 que al ubicarlo en la escala de valoración previamente definida cae en la zona de "Expectativa de FAING adecuada"; el valor medio de las percepciones fue de 73,40 que implica que cae en la zona de "Percepción de FAING adecuada".

En la figura $\mathrm{N}^{\circ} 8$ se aprecia el comportamiento global de las expectativas y percepciones del estudiante considerando la Escuela Profesional, concluyendo que es la EP de Ing. Agroindustrial en donde existe un mayor nivel de insatisfacción.

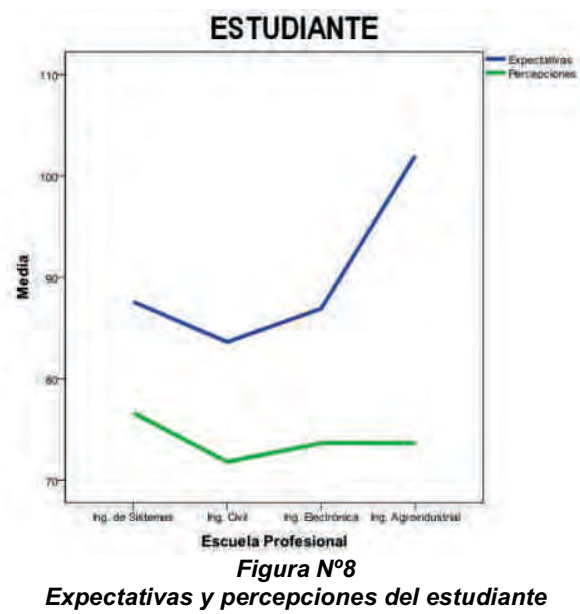

En la figura $\mathrm{N}^{\circ} 9$ se aprecia el comportamiento de cada una de las respuestas globales obtenidas referidas a la satisfacción 0 insatisfacción estudiantil con una media de 13.64 y desviación típica de 25.798, que implica que la media cae debajo de la zona de "Expectativa (Percepción) de FAING muy inadecuada".

Se concluye por tanto, que existe un nivel de insatisfacción estudiantil por la calidad de la enseñanza recibida en la FAING - UPT, a pesar de que consideran que el nivel educativo es adecuado, concluyendo que es la EP de Ing. Agroindustrial en donde existe un mayor nivel de insatisfacción. 


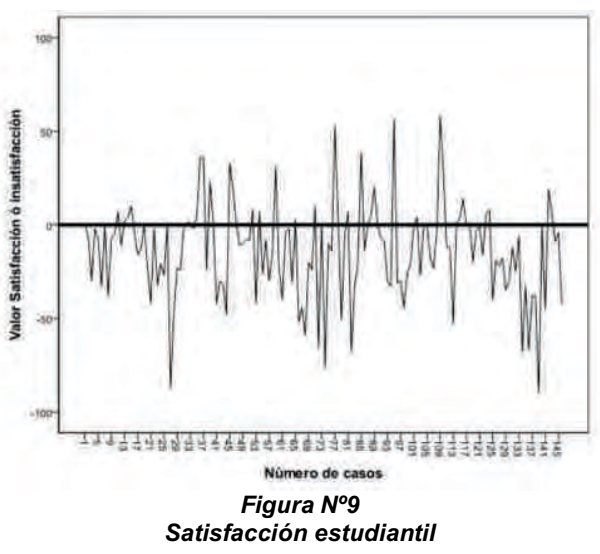

Discusión

La hipótesis general planteada indica que "El modelo educativo propuesto de gestión de calidad educativa permitirá mejorar la satisfacción estudiantil en la Facultad de Ingeniería de la Universidad Privada de Tacna". En base al análisis desarrollado se encontró que habría que elevar el nivel de satisfacción estudiantil por lo cual se efectúa una propuesta de Modelo Educativo de gestión de calidad educativa, en el cual se desarrolla, de forma detallada, los pasos que deberían implementar las Escuelas Profesionales de la Facultad de Ingeniería de la Universidad Privada de Tacna con la finalidad de proporcionar un mejor servicio educativo que permita optimizar el nivel de satisfacción estudiantil.

\section{Referencias bibliográficas}

1. REY GARCÍA, Amalio Alejandro. (1998). "Cómo gestionar la calidad en las universidades". Universidad Católica de Madrid (España).

2. RUIZ-OLALLA, C. (2001). "Gestión de la calidad del servicio". [en línea] 5campus.com. Control de Gestión $<$ http://www.5campus.com/leccion/calidads erv> [citado 1 Noviembre 2002].

3. SÁNCHEZ, M. (2001). "Calidad Total. Organizaciones de calidad, organizaciones de éxito". Ediciones Libertarias. Madrid (España).

4. Modelo ISO 9001-2008.

5. Modelo de calidad Deming. www.deming.org
6. Modelo de calidad EFQM. www.efqm.org

7. Artículo comparación de modelos de calidad

http://www.rebiun.org/opencms/opencms/ha ndle404?exporturi=/export/docReb/efqm_co mparacion.pdf\&\%5d, Comparación de modelos de calidad.

8. Modelo de calidad Baldrige. http://www.baldrige.com/

9. El modelo EFQM de Excelencia (2010), Enric Brull Alabart.

http://webfacil.tinet.org/usuaris/ebrull/CdGEFQM_2010_20110212195120.pdf.

10. Mejora continua de la calidad en los procesos. Manual García, Carlos Quispe, Luis Páez.

http://sisbib.unmsm.edu.pe/bibvirtualdata/pu blicaciones/indata/Vol6_n1/pdf/mejora.pdf.

Recibido: 26/09/13

Aceptado para publicación: 13/11/2013 